\title{
Respuesta agronómica de tres variedades de brachiaria en el cantón El Empalme provincia del Guayas, Ecuador
}

\section{Agronomic response of three varieties of brachiaria in canton El Empalme Guayas province, Ecuador}

\author{
${ }^{\triangle}$ Ricardo Augusto Luna Murillo ${ }^{1}$, Juan José Reyes Pérez¹, Juan Humberto Avellaneda Cevallos², \\ Ana Lucia Espinoza Coronel ${ }^{3}$, Nancy Bárbara Iza Taco ${ }^{3}$, Marcelo Vicente Luna Murillo ${ }^{4}$ \\ ${ }^{1}$ Universidad Técnica del Cotopaxi Extensión La Mana, Avenida Los Almendros y Pujili. Edificio Universitario. \\ La Maná,Ecuador. ${ }^{\circ}$ ricardo.luna@utc.edu.ec; jjreyesp1981@gmail.com \\ ${ }^{2}$ Facultad de Ciencias Pecuarias, Campus Finca Experimental "La Maria” km 7 vía Quevedo-El Empalme. Universidad \\ Técnica Estatal de Quevedo.EC.120501.Quevedo,Ecuador. javellaneda@uteq.edu.ec \\ ${ }^{3}$ Consultoría técnica, ConsultAR, Parroquia El Guayacán Lotización la Carmela, manzana G solar 14. consultar_ar@yahoo.es \\ ${ }^{4}$ Universidad Estatal Amazónica. Campus principal Km 2 1/2 vía Tena (Paso Lateral). mluna@uea.edu.ec
}

\section{Resumen}

$\mathrm{E}^{1}$ presente trabajo se desarrolló con el objetivo de evaluar la respuesta agronómica de tres variedades de pastos del género Brachiaria ( $B$. decumbens; $B$. brizantha y $B$. híbrido cv. Mulato (CIAT 36061), como contribución al mejoramiento de la producción animal en el Ecuador. Se empleó un diseño de bloque al azar en arreglo factorial $(2 \times 3)$ con tres repeticiones, con un total de 54 parcelas. Se evaluaron dos localidades y tres estados de madurez $(21 ; 42 ;$ y 63 días). Las variables evaluadas fueron altura de planta, número de hojas y de tallos, relación hoja:tallo y biomasa forrajera. Las variedades estudiadas tuvieron un comportamiento superior en la localidad La Guayas, en relación al suelo ácido característico de la zona. El pasto $B$. mulato se mostró superior en la producción de follaje al resto de las variedades. A los 63 días se observaron los mayores valores en altura de planta $(98.57 \mathrm{~cm})$, número de hojas (774.84) y de tallos (162.51). El rendimiento peso de hoja (6.48 t MS/ha ${ }^{-1}$ ) resultó superior a los 42 días. La producción de biomasa evidenció un incremento del rendimiento al aumentar la edad de rebrote. Se concluye que en la localidad La Guayas las especies de Brachiaria estudiadas tuvieron un desempeño superior, sobresaliendo la $B$. mulato, principalmente en el rendimiento. La edad de corte tuvo un marcado efecto en el comportamiento de los indicadores evaluados al aumentar el rendimiento.

Palabras clave: brachiaria, comportamiento agronómico, edad de corte, forrajes, pastos.

Recibido: 27-octubre-2014. Recibido en forma corregida: 25-junio-2015. Aceptado: 30-octubre-2015.

Publicado como NOTA TÉCNICA en Ciencia y Tecnología 8(2): 45-50

Diciembre de 2015

\begin{abstract}
$\mathrm{n}$ this paper, the agronomic response of three varieties Iof Brachiaria (B. decumbens; B. brizantha and $B$. hybrid cultivar cv. Mulato (CIAT 36061) was evaluated, as a contribution to improving animal production in Ecuador. A randomized block design in factorial arrangement with three replications was conducted, with a total of 54 plots. Two localities and three stages of maturity $(21 ; 42 ; 21$ and 63$)$ were evaluated. The variables evaluated were plant height, number of leaves and stems, leaf:stem and forage biomass. The varieties studied had superior performance in the town's Guayas, in relation to the characteristic acidic of soil in the area. B. mulatto grass was superior in producing foliage to other varieties. At 63 days, the highest values were observed in plant height $(98.57 \mathrm{~cm})$, number of leaves (774.84) and stems (162.51). The weight of leaf (6.48 $\mathrm{t} \mathrm{MS} / \mathrm{ha}^{-1}$ ) yield was higher at 42 days. Biomass production showed a yield increase with increasing age of regrowth. It is concluded that in the town's Guayas Brachiaria species studied had superior performance, excelling $B$. mulatto, mainly in performance. The age cutoff had a marked effect on the behavior of the indicators evaluated to increase performance.
\end{abstract}

Key words: brachiaria, agronomic performance, cutting age, fodder, pastures. 


\section{Introducción}

E n el trópico la principal fuente de nutrientes y la alimentación más barata para el ganado vacuno son los pastos y forrajes, que apoya en su economía y en la no competencia con las necesidades de alimentos para el consumo humano directo y de otros animales (Díaz, 2001).

A pesar de ello, la disponibilidad de pastos y forrajes de buena calidad ha sido una de las principales limitaciones en los trópicos. En los últimos 25 años el género Brachiaria ha tenido importancia debido a su impacto económico, llegando a ocupar cientos de hectáreas en el Ecuador, dicha importancia económica se debe en parte a su exitoso establecimiento en áreas degradadas (Baque y Tuarez, 2010), constituyendo una opción para asegurar la sostenibilidad alimentaria y así potenciar la ganadería en la región tropical (RojasHernández et al., 2011).

El género Brachiaria posee especies consideradas de importancia, se destacan la $B$. purpurascens, $B$. decumbens, $B$. humidicola y $B$. dictyoneura, por las buenas cualidades de adaptación y persistencia en suelos con limitantes, como los suelos ácidos, los suelos bajos y los de mediana y baja fertilidad; por su eficiente crecimiento y perdurabilidad; por sus altas producciones de biomasa de buena calidad y su alto grado de aceptación por los animales (Olivera et al., 2006).

En tal sentido evaluar la dinámica del crecimiento y el momento óptimo para utilizar especies forrajeras en determinadas regiones y bajo diferentes tipologías de suelo, constituyen una práctica que puede generar recomendaciones más acertadas a los productores para la elección de especies con mayores potencialidades (Sosa et al., 2008).

Por tanto, el objetivo de este estudio fue evaluar la respuesta agronómica de tres especies del género Brachiaria, para contribuir al mejoramiento de la producción animal en el Ecuador.

\section{Materiales y métodos}

T a investigación se desarrolló durante tres meses

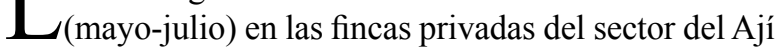
de la parroquia, La Guayas en el cantón El Empalme y en la vía El Empalme-Balzar, localizadas geográficamente a $01^{\circ} 06^{\prime}$ de latitud sur y $79^{\circ} 29$ de longitud oeste a una altura de $73 \mathrm{msnm}$. La zona ecológica corresponde a un bosque húmedo tropical, con temperatura $25.80^{\circ} \mathrm{C}$; humedad relativa promedio $86 \%$; precipitación media $1890.10 \mathrm{~mm} /$ año.

La características químicas del suelo se presentan en el Cuadro 1. Se utilizaron 54 parcelas experimentales de $1 \mathrm{~m} \times 2 \mathrm{~m}$, la siembra de las variedades se realizó utilizando cepas de plantas maduras, espaciadas a 50 $\mathrm{cm}$ entre calle y $50 \mathrm{~cm}$ entre planta. Al momento de la siembra se fertilizó con NPK (1250 kg ha-1). Además se realizaron los controles de plantas indeseables de acuerdo a su presencia.

\begin{tabular}{|c|c|c|}
\hline Componentes & El Empalme & La Guayas \\
\hline $\mathrm{pH}$ & 6.20 Ligero Acido & 5.47 Ácido \\
\hline M.O & 6.30 Alto & 4.88 Medio \\
\hline NH4 ppm & 15.00 Bajo & 31.61 Medio \\
\hline P ppm & 51.00 Alto & 27.87 Alto \\
\hline S ppm & 12.00 Medio & 14.50 Alto \\
\hline $\mathrm{K}$ meq/100 mL & 0.54 Alto & 1.62 Alto \\
\hline Ca meq/100 mL & 15.00 Alto & 12.00 Alto \\
\hline $\mathrm{Mg}$ meq/100 mL & 0.80 Bajo & 2.30 Medio \\
\hline Fe ppm & 125.00 Alto & 278.00 Alto \\
\hline Zn ppm & 11.80 Alto & 14.50 Alto \\
\hline Mn ppm & 7.30 Medio & 28.40 Alto \\
\hline $\mathrm{Ca} / \mathrm{Mg}$ & 18.70 & 5.22 \\
\hline $\mathrm{Mg} / \mathrm{K}$ & 1.48 & 1.42 \\
\hline$(\mathrm{Ca}+\mathrm{Mg}) / \mathrm{k}$ & 29.26 & 8.83 \\
\hline
\end{tabular}

Fuente: INIAP (2014) y AGROLAB (2014). 
Se utilizaron tres especies de Brachiaria ( $B$ decumbens; $B$. brizantha y la B. híbrido cv. Mulato (CIAT 36061). Al inicio de la evaluación se realizó un corte de uniformidad a $10 \mathrm{~cm}$ del suelo. Se utilizó un diseño de bloques al azar (DBCA) en arreglo factorial con tres repeticiones, en base a las localidades, las tres variedades de Brachiaria y los tres estados de madurez (21; 42; y 63 días).

La altura de planta $(\mathrm{cm})$ se obtuvo al medir con una cinta métrica desde el nivel del suelo hasta el inicio del último brote a cinco plantas recolectadas al azar dentro de cada parcela por tratamiento (unidad experimental) y frecuencia de corte. El número de hojas y número de tallos se determinó al contar todas las hojas y tallos que poseían las cinco plantas de cada unidad experimental, luego se registró el valor promedio. El rendimiento $(\mathrm{t}$ $\left.\mathrm{MS} / \mathrm{ha}^{-1}\right)$, se calculó considerando el peso seco de hojas y tallos luego de secados en la estufa de aire forzado a 65 ${ }^{\circ} \mathrm{C}$ por 48 horas. Para determinar la relación hoja-tallo se pesó con una balanza analítica, de forma individual las hojas y tallos secados en la estufa.

Para la comparación entre las medias de los tratamientos se realizaron análisis de varianza y comparaciones múltiples de medias (Tukey, $\mathrm{p}<0.05$ ). Los análisis estadísticos se realizaron con el programa Statistic v. 10.0 para Windows (StatSoft, Inc., 2011).

El modelo matemático es: $Y \mathrm{ij}=\mu+\tau \mathrm{i}+\beta \mathrm{j}+\varepsilon \mathrm{ij}$ de donde: $\mu=$ Parámetro, efecto medio; $\tau \mathrm{i}=$ Parámetro, efecto del tratamiento; $\beta \mathrm{j}=$ Parámetro, efecto del bloque; y $\varepsilon \mathrm{ij}=$ Valor aleatorio, error experimental de la u.e. i,j y Yij = Observación en la unidad experimental.

\section{Resultados y discusión}

\section{Efecto de la localidad}

El efecto de localidades en el comportamiento agronómico de especies de pasto Brachiaria se resume en el Cuadro 2, donde se puede observar que el Cantón La Guayas, las especies estudiadas, tuvieron un mejor comportamiento, evidenciado en casi la totalidad de los indicadores evaluados con mayor destaque para el rendimiento de biomasa forrajera con $3.07 \mathrm{t}$ MS ha${ }^{1}$, superior en $1.20 \mathrm{t} \mathrm{MS} \mathrm{ha}^{-1}$ respecto a la localidad El Empalme. Lo cual pudiera estar relacionado al suelo ácido (Cuadro 1) presente en el Cantón La Guayas. reportes indican que la mayoría de las especies comerciales de Brachiaria se adaptan a una amplia variedad de tipos de suelo y su desempeño es mucho mejor en los suelos ácidos (Thomas y Grof., 1986; Paulino et al., 1987; Paulino et al., 1991 y Renvoize et al., 1998).

\section{Efecto de especie}

Entre las variedades de Brachiaria estudiadas se verificó (Cuadro 3) que la $B$. brizantha fue ligeramente superior en altura $(71.64 \mathrm{~cm})$ respecto a la $B$ decumbens $(67.83 \mathrm{~cm})$ y $B$. mulato $(71.02 \mathrm{~cm})$. Al respecto, Avellaneda et al. (2008) reportan que la $B$. brizantha fue superior en altura a la decumbens y mulato, en el cantón Mocache, provincia de Los Ríos, Ecuador. De igual, en los estudios realizados por Pérez et al. (1997), se observó que la $B$. brizantha, presentó mayor altura al compararla con otras especies del mismo género. La altura promedio de la $B$. brizantha $(71.64 \mathrm{~cm})$ y $B$. decumbens $(67.83 \mathrm{~cm})$ resultó superior a la reportada por González et al. (1997) quienes evaluaron los mismos en la Amazonía ecuatoriana con valores de 70.00 y 64.30 $\mathrm{cm}$, respectivamente.

El rendimiento (t MSn ha ${ }^{-1}$ ) (Cuadro 3) mostró un mejor comportamiento en el pasto B. mulato con (2.08), en relación con decumbens (1.24) y brizantha (1.76), valores que superan a los obtenidos por Rojas-Hernández et al. (2011) al estudiar el comportamiento agronómico de Brachiarias en el trópico de Guerrero, México con valores de $1.10 \mathrm{t} \mathrm{MS} \mathrm{ha}^{-1}$ en el hibrido B. mulato.

\begin{tabular}{|c|c|c|c|}
\hline \multirow{2}{*}{ Variables } & \multicolumn{2}{|c|}{ Localidades } & \multirow{2}{*}{ EEM } \\
\hline & El Empalme & La Guayas & \\
\hline Altura de planta $(\mathrm{cm})$ & $71.48 \mathrm{a}$ & $68.77 \mathrm{a}$ & 5.63 \\
\hline Peso de hoja (kg MS ha-1) & $1.73 \mathrm{~b}$ & $9.74 \mathrm{a}$ & 3.23 \\
\hline Peso de tallo (kg MS ha $\left.{ }^{-1}\right)$ & $8.63 \mathrm{~b}$ & $8.91 \mathrm{a}$ & 0.75 \\
\hline Número de hojas & $729.85 \mathrm{a}$ & $243.53 \mathrm{~b}$ & 0.99 \\
\hline Número de tallos & $151.16 \mathrm{a}$ & $53.88 \mathrm{~b}$ & 4.68 \\
\hline Relación hoja: tallo & $2.5 \mathrm{a}$ & $1.17 \mathrm{~b}$ & 0.75 \\
\hline Biomasa (t MS ha-1) & $1.87 \mathrm{~b}$ & $3.07 \mathrm{a}$ & 3.42 \\
\hline
\end{tabular}

Promedios con letras iguales no difieren estadísticamente, según Tukey ( $\mathrm{p}>0.05)$ 
Luna et al., 2015

Cuadro 3. Efecto de variedades en el comportamiento agronómico de variedades de Brachiaria

\begin{tabular}{lrrrr}
\hline \multirow{2}{*}{ Variables } & \multicolumn{3}{c}{ Brachiaria } & \multirow{2}{*}{ EEM } \\
\cline { 2 - 4 } & B. decumbens & B. brizantha & B. mulato & \\
\hline Altura de planta (cm) & $67.83 \mathrm{a}$ & $71.64 \mathrm{a}$ & $71.02 \mathrm{a}$ & 6.97 \\
Peso de hoja (t MS ha $\left.{ }^{-1}\right)$ & $4.62 \mathrm{~b}$ & $3.94 \mathrm{~b}$ & $8.64 \mathrm{a}$ & 4.93 \\
Peso de tallo $\left(\mathrm{t} \mathrm{MS} \mathrm{ha}^{-1}\right)$ & $5.10 \mathrm{ab}$ & $3.19 \mathrm{~b}$ & $6.35 \mathrm{a}$ & 1.05 \\
Número de hojas & $634.48 \mathrm{a}$ & $337.2 \mathrm{~b}$ & $502.8 \mathrm{ab}$ & 1.20 \\
Número de tallos & $148.80 \mathrm{a}$ & $63.12 \mathrm{~b}$ & $98.11 \mathrm{ab}$ & 6.61 \\
Relación hoja: tallo & $1.22 \mathrm{~b}$ & $1.94 \mathrm{a}$ & $2.42 \mathrm{a}$ & 1.05 \\
Biomasa (t MS/ha & $1.24 \mathrm{~b}$ & $1.76 \mathrm{~b}$ & $2.08 \mathrm{a}$ & 4.78 \\
\hline
\end{tabular}

Promedios con letras iguales no difieren estadísticamente, según Tukey ( $\mathrm{p} \geq 0.05)$

El comportamiento observado de los cultivares de Brachiaria estudiados coincide los resultados obtenidos por Guenni et al. (2005) quienes indican que entre varias accesiones de Brachiaria el pasto mulato se adaptó mejor que el resto de los cultivares, reflejado en su mayor acumulación de material vivo.

\section{Efecto de la edad de la planta}

A los 63 días (Cuadro 4) se observaron los mayores valores en altura de planta $(98.57 \mathrm{~cm})$, número de hojas (774.84) y número de tallos (162.51), coincidiendo con lo referido por Newman y Delgado (1999), que el número de hojas estará siempre por encima del número de tallos a partir del día 28, explicando una calidad nutritiva mayor en los primeros estadios de la planta. Autores como Vega et al. (2006) y Ramírez et al. (2009), consideran que la edad de rebrote constituye uno de los factores de mayor influencia en el crecimiento y la calidad de los pastos, a medida que se prolonga la edad de rebrote se logra rendimiento superior, con deterioro de la calidad; esto indica que defoliaciones frecuentes son a menudo más deseables para utilizar pasto de mayor valor nutritivo.

La respuesta observada en las variedades de pastos analizadas en cuanto a incrementar la producción de biomasa con la edad de rebrote, coincide con los reportes de García et al. (2008); Verdecia et al. (2008); Ramírez et al. (2009); Reyes et al. (2009) y Fernández et al. (2012) quienes evaluaron diferentes variedades de pastos del género Brachiaria y Panicum en las condiciones edafoclimáticas de la provincia Granma, aspectos que están relacionados a la mayor cantidad de componentes de la pared celular y reducción de los contenidos celulares (Chacón et al., 2009; Valenciaga et al., 2009; Ojeda et al., 2010). Asi mismo, Lemaire (2001); Fernández et al. (2004) y Velasco-Zebadúa et al. (2007) explican el efecto del estímulo del corte en la producción de MS de los pastos. Efecto de las

Cuadro 4. Efecto de estados de madurez en el comportamiento agronómico de variedades de Brachiaria

\begin{tabular}{|c|c|c|c|c|}
\hline \multirow{2}{*}{ Variables } & \multicolumn{3}{|c|}{ Estados de madurez (días) } & \multirow{2}{*}{ EEM } \\
\hline & 21 & 42 & 63 & \\
\hline Altura de planta $(\mathrm{cm})$ & $53.22 \mathrm{~b}$ & $57.71 \mathrm{~b}$ & 98.57 a & a 4.79 \\
\hline Peso de hoja (t MS ha-1) & $9.50 \mathrm{a}$ & $9.93 \mathrm{a}$ & $6.48 \mathrm{~b}$ & b $\quad 4.22$ \\
\hline Peso de tallo (t MS ha-1) & $5.03 \mathrm{a}$ & $4.65 \mathrm{a}$ & $5.01 \mathrm{a}$ & a $\quad 0.90$ \\
\hline Número de hojas & $227.02 \mathrm{c}$ & $457.29 \mathrm{~b}$ & 774.84 a & a 1.06 \\
\hline Número de tallos & $61.01 \mathrm{c}$ & $84.44 \mathrm{~b}$ & $162.51 \mathrm{a}$ & a 5.66 \\
\hline Relación hoja: tallo & $2.05 \mathrm{a}$ & $1.90 \mathrm{a}$ & $1.61 \mathrm{a}$ & a $\quad 0.90$ \\
\hline Biomasa (t MS/ha/corte) & $0.48 \mathrm{~b}$ & $0.69 \mathrm{~b}$ & $1.24 \mathrm{a}$ & $\mathrm{a} \quad 4.05$ \\
\hline
\end{tabular}

Promedios con letras iguales no difieren estadísticamente, según Tukey ( $\mathrm{p} \geq 0.05)$ 
interacciones entre localidades por variedades por estados de madurez

En la localidad La Guayas, las especies estudiadas tuvieron un comportamiento superior (Figura 1) lo cual ya ha sido descrito, mucho más evidente a partir de los 63 días de corte. Puede observarse que existe una fuerte interacción a los 42 días en la altura de planta, entre la localidad El Empalme y La Guayas, con respecto al pasto $B$. decumbens (Figura 1A). Igualmente hay interacción en los valores de altura de planta del pasto mulato a los 63 días con 99.60 y $100.53 \mathrm{~cm}$ respectivamente en ambas localidades.

El rendimiento es una importante variable para conocer el nivel productivo de los pastos, en este estudio se evidenció un incremento del rendimiento al aumentar la edad de rebrote (Figura 1B). Autores como Beneval y Siqueira (1987) reportan un incremento del rendimeinto de B. ruziziensis y $B$. decumbens a medida que se incrementa la edad de corte, aspectos antes señalados en este estudio.

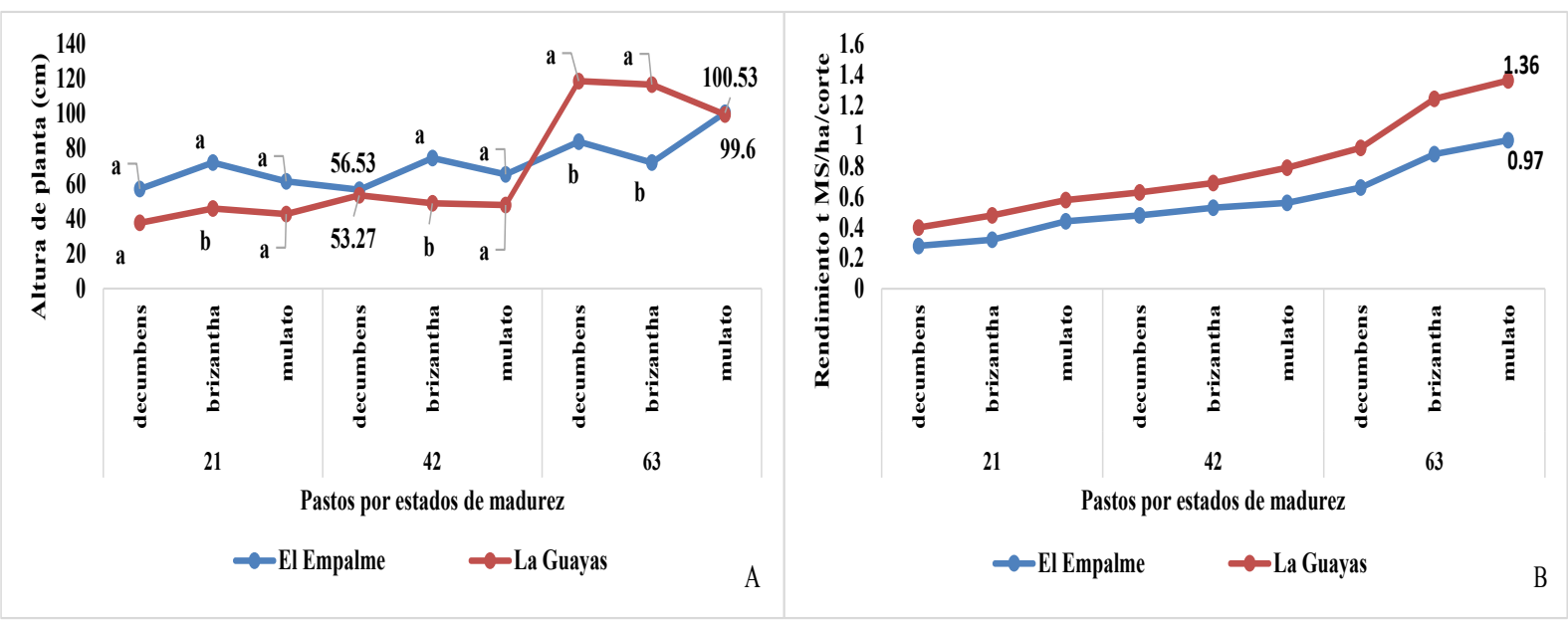

Figura 1. Efecto del estado de madurez en el comportamiento agronómico de variedades de Brachiaria en las localidades El Empalme y La Guayas. Altura de Planta (A), Rendimiento (B)

\section{Conclusiones}

$\mathrm{E}^{\mathrm{n}}$ la localidad La Guayas las especies de Brachiaria estudiadas tuvieron un desempeño superior, sobresaliendo la $B$. mulato principalmente en la producción de biomasa. La edad de corte tuvo un marcado efecto en el comportamiento de los indicadores evaluados.

\section{Bibliografía}

AGROLAB. 2014. Laboratorio de Análisis Químico Agropecuario. Universidad de Cotopaxi. Ecuador.

Avellaneda, C.J., Cabezas, G.F., Quintana, Z.G., Luna, M.R., Montañez, V.O., Espinoza, G.I., Zambrano, M.S., Romero, G.D., Vanegas, R.J., Pinargote, M.E. 2008. Comportamiento agronómico y composición química de tres variedades de Brachiaria en diferentes edades de cosecha. Ciencia y Tecnología 1(2): 87-94.

Baque, M., Tuarez, V. 2010. Comportamiento Agronómico y valor nutritivo de diez variedades de pastos en diferentes estados de madurez, en la parroquia la Guayas del cantón El Empalme. Universidad Técnica Estatal de Quevedo, Ecuador. $80 \mathrm{p}$.

Beneval, R., Siqueira, B.A.J. 1987. Composiçao química e rendimento do capim andropogon (Andropogon gayanus var. Bisquamulatus cv. Planaltina) em diferentes idades de corte. Anais das Escolas de Agronomia e de Veterinária 17: 49-58.

Chacón, P., Vargas, C. 2009. Digestibilidad y calidad del Pennisetum purpureum cv. king grass a tres edades de rebrote. Agronomía Mesoamericana, 20(2): 399-408.

Díaz, A. 2001. Producción de biomasa de (Eichhornia crassipes) en aguas residuales porcinas. Tesis en opción al título de Master en Nutrición Animal. Universidad de Granma, Cuba.

Fernández, J.L., Benítez, D.E., Gómez, I., de Souza, A., Espinosa, R. 2004. Rendimiento de MS y contenido de proteína bruta del pasto Panicum maximum vc likoni en un suelo vertisol de la provincia Granma. Revista Cubana de Ciencia Agrícola 38(4): 417421. 
García, R.O., Martínez, R., Tuero, A., Cruz, M., Romero, A., Estanquero, L., Noda, A., Torres, V. 2008. Evaluación Agronómica de guinea mombasa (Panicum maximum Jacq.) en un suelo ferralitico rojo típico de la provincia La Habana. Revista Cubana de Ciencia Agrícola 42(2): 205-208.

González, M.R., Anzúlez, S.A., Vera, Z.A., Riera, B.L. 1997. Manual de pastos para la amazonia ecuatoriana. INIAP. Ecuador.

Guenni, O., Gil, J.L., Guedez, Y. 2005. Growth, forage yield and light interception and use by stands of five Brachiaria species in a tropical environment. Tropical Grasslands. 39: 42-53.

INIAP. 2014. Laboratorio de suelos, tejidos vegetales y aguas (INIAP - Pichilingue). Quevedo. Ecuador.

Lemaire, G. 2001. Ecophysiology of grassland. Aspects of forage plant populations in grazed swards. In: Proc. XIX Internatl. GRassland Congreso. Brazil Soc. Animal Husbandry. Sao Pedro, Sao Paulo Brazil. 29-37 p.

Newman, Y., Delgado, H. 1999. Crecimiento del pasto buffel. 1. Arquitectura de planta. Revista de la Facultad de Agronomía de la Universidad del Zulia 16: 319-326.

Ojeda, F., Bárbara, N.P., Lamela, L., Santana, H. y Montejo, I. 2010. Estudio de un ciclo de ceba con una dieta integral a base de forraje hollejo de cítricos. 1. Efecto de la calidad del forraje. Pastos y Forrajes 33(1): 81.

Olivera, Y., Machado, R., del Pozo, P.P. 2006 Características botánicas y agronómicas de especies forrajeras importantes del género Brachiaria. Pastos y Forrajes 29(1): 5-29.

Paulino, V., Anton, D., Colozza, M. 1987. Problemas nutricionais do género Brachiaria e algunas relaçǒes com o comportamento animal. Zootecnia, 25: 215-263.

Paulino, V., Pedreira, J., Camargo, D., Meirelles, N., Bianchini, D., Oliveira, P. (eds). 1991. Memorias del $2^{\circ}$ encontró para discussao sobre capins do género Brachiaria. Instituto de Zootecnia y Fundaçao de Amparo á Pesquisa do Estado de Sao Paulo (FAPSEP), 45-135 pp.

Pérez, S., Faria, M., González, B. 1997. Evaluación agronómica de gramíneas forrajeras en Carora, Estado Lara, Venezuela. Facultad de Agronomía de la Universidad del Zulia 16: 621-636.

Ramírez, O., Hernández, A., Carneiro, S., Pérez, J., Enríquez, J.F., Quero, A., Guadalupe, J., Herrera, H., Cervantes, A. 2009. Acumulación de forraje, crecimiento y características del pasto mombasa (Panicum maximum Jacq.). Revista Técnica Pecuaria, 47(2): 203-213.

Renvoize SA, Clayton WD, Kabuye CHS. 1998.
Morfología, taxonomía y distribución natural de Brachiaria (Trin.) Griseb. En: Brachiaria: Biología, Agronomía y Mejoramiento. Miles J.W., B.L. Maass y C.B do Valle (Eds.). CIATEMBRAPA/ CNPGC, Cali. 1-17 p.

Reyes-Purata, A., Bolaños-Aguilar, E.D., HernándezSánchez, D., Aranda-lbañez, E.M., IzquierdoReyes, F. 2009. Producción de materia seca y concentración de proteína en 21 genotipos del pasto humidícola Brachiaria humidicola (Rendle) Schweick. Universidad y Ciencia 25(3): 213-224.

Rojas-Hernández, S., Olivares-Pérez, J., JiménezGuillén, R., Gutiérrez-Segura, I., Avilés-Nova, F. 2011. Producción de materia seca y componentes morfológicos de cuatro cultivares de Brachiaria en el trópico. Avances en Investigación Agropecuaria, 15(1): 3-8.

Sosa, R.E.E., Cabrera, T.E., Pérez, R.D., Ortega, R.L. 2008. Producción estacional de materia seca de gramíneas y leguminosas forrajeras con cortes en el estado de Quintana. Revista Técnica Pecuaria de México 46(004): 413-426.

StatSoft Inc. 2011. Statistica.System reference. StatSoft, Inc., Tulsa, Oklahoma, USA. 1098 p.

Thomas, D., Grof, B. 1986. Some pasture species for the tropical savannas of South America. III: Andropogon gayanus, Brachiaria spp. and Panicum maximum. In Herbage Abstracts, 56(12): 557-565.

Vega, E., Ramírez, J.L., Leonard, I., Igarza, A. 2006. Rendimiento, caracterización química y digestibilidad del pasto Brachiaria decumbens en las actuales condiciones edafoclimáticas del Valle del Cauto. REDVET, Revista Electrónica de Veterinaria 7(5): 1-6.

Velasco-Zebadúa, M.E., Hernández-Garay, A., González-Hernández, V.A. 2007. Cambios en componentes del rendimiento de una pradera de ballico perenne, en frecuenta a la respuesta de corte. Revista Fitotecnia Mexicana 30(1): 79-87.

Verdecia, D., Ramírez, J.L., Leonard, I., Pascual, Y., López, Y. 2008. Rendimiento y componentes del valor nutritivo del Panicum maximum cv. Tanzania. REDVET. Revista Electrónica de Veterinaria 5: $1-9$

Vergara-López, J., Araujo-Febres, O. 2006. Producción, composición química y degradabilidad ruminal in situ de Brachiaria humidicola (Rendle) Schweick en el bosque seco tropical. Revista Científica 16 (3): 239-248.

Valenciaga, D., Chongo, B., Oramas, A. 2009. Efecto de la edad de rebrote en la composición química de Pennisetum purpureum cv. CUBA CT-115. Revista Cubana de Ciencias Agrícolas. 43(1): 73-79. 